Tohoku J. Exp. Med., 2007, 213, 33-40

\title{
Expression of Angiopoietin-2 is Correlated with Vascularization and Tumor Size in Human Colorectal Adenocarcinoma
}

\author{
Hong-Ling Wang, ${ }^{1}$ Chang-Sheng Deng, ${ }^{1}$ Jun Lin,,${ }^{1}$ Ding-Yu Pan, ${ }^{2}$ Zu-Yu Zou ${ }^{3}$ \\ and XIAO-YANG Z $\mathrm{HOU}^{4}$ \\ ${ }^{1}$ Department of Gastroenterology, Zhongnan Hospital of Wuhan University, Wuhan, China \\ ${ }^{2}$ Department of General Surgery, Zhongnan Hospital of Wuhan University, Wuhan, China \\ ${ }^{3}$ Department of Pathology, Zhongnan Hospital of Wuhan University, Wuhan, China \\ ${ }^{4}$ Department of Cardiology, Renmin Hospital of Wuhan University, Wuhan, China
}

\begin{abstract}
Wang, H.-L., Deng, C.-S., Lin, J., Pan, D.-Y., Zou, Z.-Y. and Zhou, X.-Y. Expression of Angiopoietin-2 is Correlated with Vascularization and Tumor Size in Human Colorectal Adenocarcinoma. Tohoku J. Exp. Med., 2007, 213 (1), 33-40 - Angiopoietins are endothelial growth factors, which play crucial roles in normal vascular development and tumor angiogenesis. We examined the expression profiles of angiopoietin-1 (Ang-1), angiopoietin-2 (Ang-2), vascular endothelial growth factor (VEGF), and Tie-2, a receptor for Ang-1 and Ang-2, in both colorectal adenocarcinoma and adjacent normal tissues, as judged by histology, in order to elucidate their relationships with microvascular density (MVD) and clinicopathologic properties. Higher MVD was associated with a lower degree of differentiation of colorectal adenocarcinoma. Immunohistochemical analysis revealed that the expression of Ang-2 and VEGF was significantly increased in colorectal adenocarcinoma compared to adjacent normal tissues $(p<0.01)$, and the expression of Ang-2 positively correlated with that of VEGF ( $\mathrm{r}=0.997, p<0.01)$. In contrast, the expression of Ang-1 was lower in adenocarcinoma tissues than in adjacent normal tissues $(p<0.01)$, while there was no significant difference in Tie-2 expression in both tissues. Moreover, MVD was increased in Ang-2- and VEGF-expressing adenocarcinoma tissues compared to the Ang-2- and VEGF-negative tissues, respectively $(p<0.01)$. Importantly, MVD was lower in Ang-1-expressing adenocarcinoma tissues relative to Ang-1-negative tissues $(p<0.01)$. Furthermore, expression of Ang-2 as well as VEGF was significantly up-regulated in colorectal adenocarcinoma with diameters $\geqq 5 \mathrm{~cm}$ or with lymph-node metastases $(p<0.01)$. In conclusion, the increased expression of Ang-2 and the decreased expression of Ang-1 may be responsible for blood vessel formation and rapid growth of the colorectal adenocarcinoma. —— angiopoietins; colorectal adenocarcinoma; microvascular density; clinicopathology

(C) 2007 Tohoku University Medical Press
\end{abstract}

Received May 14, 2007; revision accepted for publication July 19, 2007.

Correspondence: Chang-Sheng Deng, Department of Gastroenterology, Zhongnan Hospital, Wuhan University, Wuhan 430071, Hubei Province, China.

e-mail: zhnwhl@yahoo.com.cn 
Colorectal cancer is one of the most common malignancies worldwide, second only to lung cancer as a cause of cancer death. Angiogenesis, the formation of new blood vessels, has been well recognized to be essential for tumor growth and expansion by supplying malignant cells with sufficient oxygen and nutrition. Moreover, it has been shown that angiogenesis is an independent prognostic factor for colorectal cancer (Saclatides and Speziale 1994).

Angiogenesis involves the activation, migration, and proliferation of endothelial cells and is regulated by several peptides and nonpeptide molecules. Of these regulators, vascular endothelial growth factor (VEGF) is a hypoxia-inducible angiogenic factor, and its up-regulation is thought to mediate many of the angiogenic effects of growth factors that are not direct endothelial cell mitogens. Therefore, VEGF is crucial for blood vessel development (Leung et al. 1989; Katoh and Katoh 2006; Carvalho et al. 2007).

Angiopoietins are members of another novel family of angiogenic factors and participate in the formation of blood vessels. Of the four currently known family members, the best characterized ones are angiopoietin-1 (Ang-1) and angiopoietin-2 (Ang-2), both of which function as ligands for the endothelium-specific tyrosine kinase receptor Tie-2 (Zadeh and Guha 2003). Ang-1 reduces endothelial permeability of noncerebral vessels and has a major role in vascular stabilization and maturation; thus Ang-1 plays an important role in maintaining vessel integrity. Ang-2, which is thought to be an endogenous antagonist of the action of Ang-1, competes for binding to the Tie-2 receptor and blocks the Ang-1-induced Tie-2 autophosphorylation during vasculogenesis, subsequently leading to loosening of cell-matrix and cell-cell contacts and allowing access to angiogenic inducers. In the absence of angiogenic growth or survival signals, blockade of Ang-1 or activation of Ang-2 may destabilize capillaries and shift the balance to endothelial cell apoptosis and a regression of vessel structures (Hanahan 1997; Yancopoulos et al. 2000). In mice and humans, Ang-2 is selectively expressed in ovary, uterus and placenta (Maisonpierre et al. 1997), while Ang-1 is widely expressed in both the embryo and the adult (Wong et al. 1997). Furthermore, recent studies provide evidence that Ang-1 and Ang-2, via competing for their receptor Tie-2, are probably involved in the regulation of vasculogenesis in both normal and tumor tissues (Zhu and Chen 2004).

Here we investigated the expression profiles of Ang-1, Ang-2, Tie-2 and VEGF in human colorectal adenocarcinoma and their relationships with microvascular density (MVD) and clinicopathologic characteristics. The results obtained suggest that these proteins may be useful to determine clinical diagnosis and prognosis of colorectal carcinoma, and may represent novel targets for cancer therapy.

\section{Materials and Methods}

\section{Clinical samples}

Surgical specimens, including cancer tissues and their adjacent normal tissues, were obtained from 45 patients with pathologically confirmed colorectal adenocarcinoma between January 2002 and December 2004 in Zhongnan Hospital of Wuhan University, China. None of the patients had undergone radiotherapy or chemotherapy prior to operation. All 45 subjects ( 29 males and 16 females) were included in this study, with a median age of 52.6 years (range 20-78 years). All resected surgical specimens were fixed with $10 \%$ buffered formalin, embedded in paraffin, and stained with hematoxylineosin for histopathologic classification. Depending on glandular architecture, cellular pleomorphism and mucosecretion of the predominant pattern, adenocarcinoma may present three degrees of differentiation: well, moderately and poorly differentiate. Furthermore, the tumors were staged according to Dukes' classification system: A, carcinoma in situ limited to mucosa or submucosa; B, cancer that extends into the muscularis, into or through the serosa; $\mathrm{C}$, cancer that extends to regional lymph nodes; and $\mathrm{D}$, cancer that has metastasized to distant sites. Our study of human materials was approved by the ethical committee of Zhongnan Hospital of Wuhan University.

\section{Reagents}

The rabbit-derived polyclonal antibodies against human Ang-1, Ang-2, Tie-2 and VEGF were purchased from Santa Cruz Biotechnology (Santa Cruz, CA, USA). 
The mouse anti-human CD34 monoclonal antibody was commercially acquired from Zhongshan Bio-company (Beijing, China). The streptavidin-peroxidase (S-P) based immunohistochemical detection kit was obtained from DAKO (Glostrup, Denmark). All other chemicals were supplied by Maixin Bio-company (Fujian, China).

\section{Immunohistochemical staining}

Paraffin sections of 4- $\mu \mathrm{m}$ thickness were deparaffinized in xylene and rehydrated, and then incubated with $3 \%$ hydrogen peroxide in methanol to block endogenous peroxidase. Each slide was incubated with normal horse serum for $20 \mathrm{~min}$ at room temperature. The primary antibody directed against Ang-1 (1:100 dilution), Ang-2 ( $1: 100$ dilution), Tie-2 ( $1: 200$ dilution) or VEGF $(1: 100$ dilution) was applied to the sections and incubated overnight at $4^{\circ} \mathrm{C}$. Sections were processed with a standard immunoperoxidase method using a streptavidin-peroxidase complex kit. The peroxidase reaction was then developed with diaminobenzidine and counterstained with hematoxylin. Human placenta was used as a positive control. For the negative control, phosphatebuffered saline (PBS) was used as a substitute for the primary antibody. Immunohistochemical staining was assessed from average luminosity and positive ratio by Automatic Magic Analysis System (Olympus BX50, Tokyo). Cells with brown-yellow or deep brown staining of the cytoplasm and plasma membrane were considered as positive cells. Because of intratumoral heterogeneity, the expression levels of Ang-1, Ang-2, Tie-2 and VEGF were graded based on corresponding positive cell ratio: negative (-) when positive cells were present in less than $20 \%$ of the area; positive (+) when positive cells were present in $20 \%$ to $50 \%$ of the area; and strongly positive $(++)$ when positive cells were present in $50 \%$ or more of the area.

\section{MVD counting}

The MVD was evaluated according to the methods described by Weidner (1995). After immuostaining with anti-CD34 monoclonal antibody ( $1: 50$ dilution), colorectal adenocarcinoma and adjacent normal tissues were first screened at a low-power magnification $(\times 40)$ to identify areas of the highest MVD. Counts were taken in 5 highest MVD areas at a high power magnification $(\times 400)$. A single blood vessel endothelial cell or cell colony stained with brown-yellow color was judged as a microvessel. The mean value of the fields counted was considered as the MVD.

\section{Statistical analysis}

All continuous variables are presented as mean \pm S.D., and comparisons between two groups or multigroup were by unpaired student's $t$-tests and ANOVA method, respectively. Categorical variables are presented as proportions, and comparisons between groups were by $\chi^{2}$ tests. The $q$-test was used for identification and rejection of outliers. In addition, the correlations between the expression of Ang-1, Ang-2, or VEGF, and MVD in colorectal adenocarcinoma were determined by analysis of linear correlation. $P<0.05$ was considered statistically significant.

\section{RESUlts}

Distribution of Ang-1, Ang-2, Tie-2 and VEGF in colorectal adenocarcinoma and adjacent normal tissues

Ang-1, Ang-2, and VEGF were expressed within the cytoplasm (Figs. 1-3). Ang-2 was highly expressed in colorectal adenocarinoma, while Ang-1 was highly expressed in normal tissues adjacent to colorectal adenocarinoma. VEGF was highly expressed in colorectal adenocarinoma tissues compared to adjacent normal tissues (Figs. $1-3)$.

Expression levels of Ang-1, Ang-2, Tie-2 and VEGF in colorectal adenocarcinoma tissues and adjacent normal tissues

In terms of average luminosity and positive ratio, the expression levels of Ang-2 and VEGF were significantly higher in colorectal adenocarcinoma than in adjacent normal tissues $(p<0.01)$. In contrast, Ang-1 was significantly down-regulated in cancer tissues compared to adjacent normal tissues $(p<0.01)$. Moreover, there was no significant difference in Tie-2 expression in both normal and cancer tissues $(p>0.05)$. It is also noteworthy that the expression of these proteins may depend upon the degree of cancer differentiation. The lower the degree of differentiation of the adenocarcinoma, the higher the expression levels of Ang-2 and VEGF; highly differentiated adenocarcinoma demonstrated higher expression of Ang-1 (Table 1). 

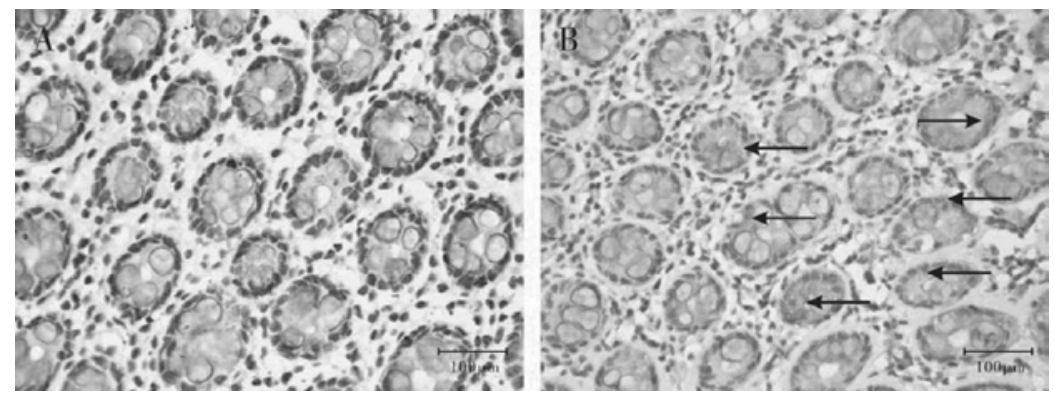

Fig. 1. Immunohistochemical staining for Ang-2 and Ang-1 in adjacent normal tissues (counterstained with hematoxylin). No positive cells were detected for Ang-2 (A), while positive staining for Ang-1 (B) was noted within the cytoplasm (arrows) (Original magnification $\times 400$ ).
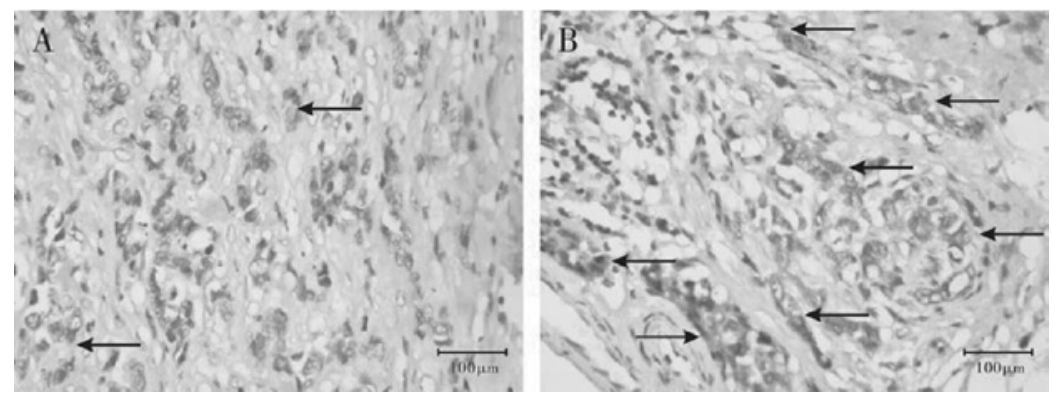

Fig. 2. Immunohistochemical staining for Ang-1 and Ang-2 in the poorly-differentiated colorectal adenocarcinoma tissues (counterstained with hematoxylin). There were few positive cells for Ang-1 (A), while many positive cells were observed for Ang-2 (B) (arrows) (Original magnification $\times 400$ ).
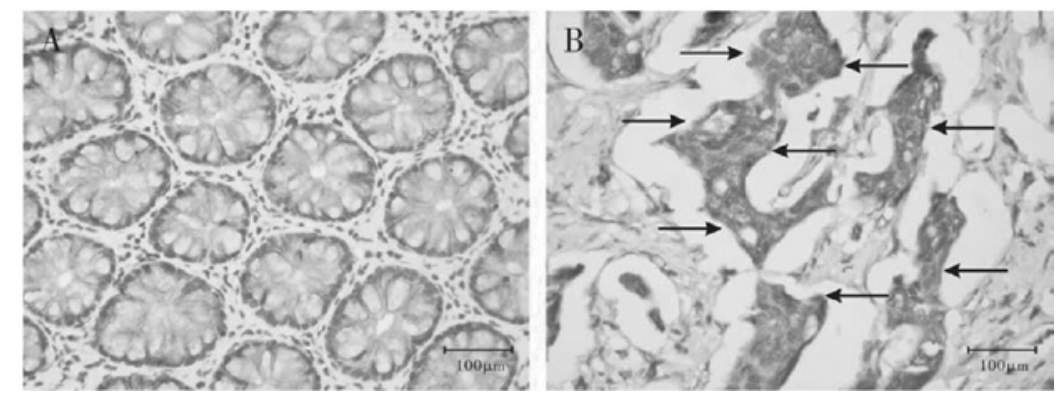

Fig. 3. Immunohistochemical staining for VEGF in both colorectal adenocarcinoma and adjacent normal tissues (counterstained with hematoxylin). There were no VEGF-positive cells in adjacent normal tissues (A), while colorectal adenocarcinoma tissues exhibited strong expression of VEGF (B) (arrows) (Original magnification $\times 400$ ).

The correlation between expression of Ang-2 and expression of VEGF in colorectal adenocarcinoma and adjacent normal tissues

As shown in Fig. 4, there was a significantly positive correlation between Ang-2 and VEGF expression in various degrees of differentiation of adenocarcinoma, as well as in their adjacent normal tissues $(r=0.997, p<0.01)$.

Comparison of MVD between colorectal adenocarcinoma and adjacent normal tissues

The MVD was significantly increased in any 
of the poorly-, moderately- and well-differentiated colorectal adenocarcinoma, compared to adjacent normal tissues. Moreover, there was an increase in MVD as the degree of differentiation of the adenocarcinoma decreased (Table 2).

The expression levels of Ang-1, Ang-2 and VEGF correlate with MVD in colorectal adenocarcinoma

The MVD in Ang-1-expressing adenocarcinoma tissues was significantly lower compared to Ang-1 negative tissues $(p<0.01)$. In contrast, it was significantly increased in Ang-2-expressing tissues than in negative tissues $(p<0.01)$. Likewise, the MVD in VEGF-expressing tissues was significantly increased compared to negative tissues $(p<0.01)$ (Table 3).

The expression levels of Ang-2 and VEGF in colorectal adenocarcinoma correlate with clinicopathologic features

As shown in Table 4, the expression of Ang-2 was associated with tumor size and lymphnode metastases, but not with the age, gender, tumor location, invasion depth or Dukes' stage. The expression of Ang-2 was significantly increased in colorectal adenocarcinoma with lymph-node metastases or in tumor $\geqq 5 \mathrm{~cm}$ in size $(p<0.01)$. Similar changes were observed for VEGF.

\section{Discussion}

Angiopoietins are regulatory factors involved in vasculogenesis. It has been recognized that angiopoietins play important roles in tumor blood vessel development (Loughna and Sato 2001). The present study was undertaken to analyze the expression profiles of Ang-1 and Ang-2 in human colorectal adenocarcinoma and adjacent normal tissues, in an effort to determine their importance in vasculogenesis and metastasis of colorectal cancer.

Our results indicate that Ang-1 and Ang-2 are expressed in both colorectal adenocarcinoma and adjacent normal tissues, but their expression levels varied between the two tissue types. In adenocarcinoma, the expression level of Ang-2 


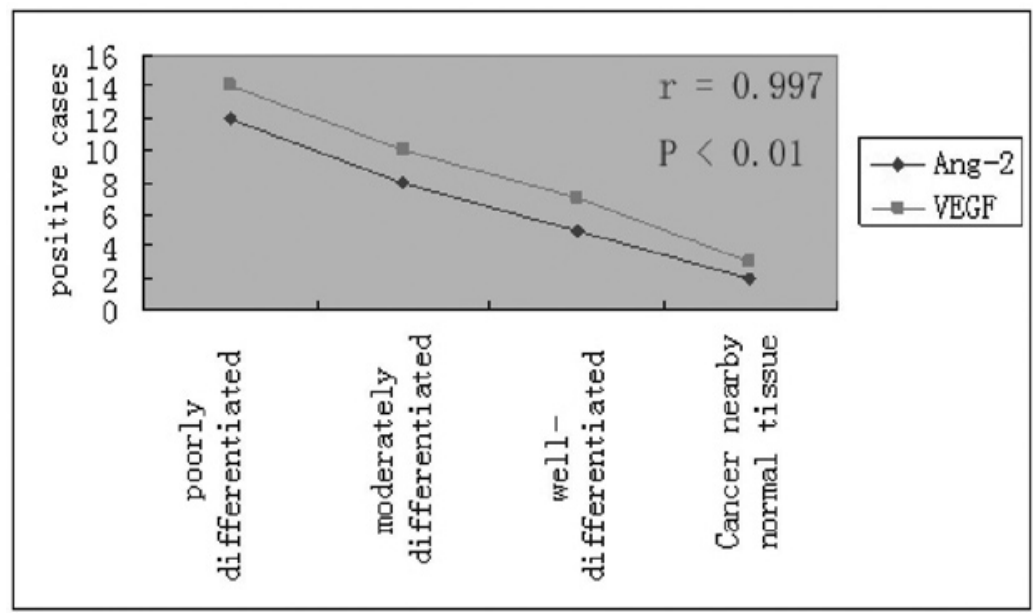

Fig. 4. The relationship between expression of Ang-2 and expression of VEGF in colorectal adenocarcinoma and adjacent normal tissues. Ang-2 showed a significant positive correlation with VEGF $(p<0.01)$.

TABLE 2. Comparison of MVD between colorectal adenocarcinoma and adjacent normal tissues (mean \pm S.D.).

\begin{tabular}{lcc}
\hline \multicolumn{1}{c}{ Group } & Case number & MVD \\
\hline Poorly-differentiated & 18 & $39.85 \pm 10.25^{* \nabla \star}$ \\
Moderately-differentiated & 16 & $21.36 \pm 9.89^{*} \triangle$ \\
Well-differentiated & 11 & $12.49 \pm 5.63^{*}$ \\
Adjacent normal tissue & 12 & $4.68 \pm 1.79$ \\
\hline
\end{tabular}

Compared with adjacent normal tissues, ${ }^{*} p<0.01$; compared with the well-differentiated tumor tissues, $\triangle p<0.05, \nabla p<0.01$; compared with the mode rately-differentiated tumor tissues, ${ }^{\star} p<0.01$.

TABle 3. The correlation between the expression of Ang-1, Ang-2 and VEGF, and MVD in colorectal adenocarcinoma (mean \pm s.D.).

\begin{tabular}{clcc}
\hline \multicolumn{2}{c}{ Subject } & Case number & MVD \\
\hline \multirow{2}{*}{ Ang-1 } & Positive & 16 & $9.82 \pm 4.66$ \\
& Negative & 29 & $28.53 \pm 10.17^{*}$ \\
\multirow{2}{*}{ Ang-2 } & Positive & 27 & $27.68 \pm 9.13$ \\
& Negative & 18 & $12.44 \pm 6.41^{\star}$ \\
\multirow{2}{*}{ VEGF } & Positive & 31 & $30.98 \pm 11.21$ \\
& Negative & 14 & $10.26 \pm 5.11^{\#}$ \\
\hline
\end{tabular}

Compared with Ang-1 positive group, ${ }^{*} p<0.01$; compared with Ang-2 positive group, ${ }^{\star} p<0.01$; compared with VEGF positive group, ${ }^{\sharp} p<0.01$.

was significantly higher compared to the normal tissues, with an increase in Ang-2 expression as the degree of differentiation decreased. In normal tissues, however, Ang-1 was predominantly expressed rather than Ang-2. Furthermore, the expression level of Ang-1 was significantly higher 
TABLE 4. The relationships between Ang-2 or VEGF expression in colorectal adenocarcinoma and clinicopathologic characteristics.

\begin{tabular}{|c|c|c|c|}
\hline Classification & Case number & $\begin{array}{l}\text { Ang-2-expression } \\
\text { rate }(\%)\end{array}$ & $\begin{array}{l}\text { VEGF-expression } \\
\text { rate }(\%)\end{array}$ \\
\hline$\geqq 60$ years & 24 & 62.5 & 70.8 \\
\hline$<60$ years & 21 & 57.1 & 66.7 \\
\hline Gender: Male & 29 & 58.6 & 72.4 \\
\hline Female & 16 & 62.5 & 62.5 \\
\hline Tumor location: Colon & 32 & 59.4 & 71.9 \\
\hline Rectum & 13 & 61.5 & 61.5 \\
\hline Tumor size: $\geqq 5 \mathrm{~cm}$ & 27 & $77.8^{\mathrm{a}}$ & $81.4^{\mathrm{c}}$ \\
\hline$<5 \mathrm{~cm}$ & 18 & 33.3 & 50.0 \\
\hline Invasion depth: Early phase & 12 & 58.3 & 66.7 \\
\hline Developed phase & 33 & 60.6 & 69.7 \\
\hline Lymph node metastases: Yes & 23 & $82.6^{\mathrm{b}}$ & $86.9^{d}$ \\
\hline \multirow{3}{*}{$\begin{aligned} & \text { Dukes' Stage: A, B } \\
& \text { C, D }\end{aligned}$} & 22 & 36.4 & 50.0 \\
\hline & 25 & 60.0 & 68.0 \\
\hline & 20 & 60.0 & 70.0 \\
\hline
\end{tabular}

Compared with tumor $<5 \mathrm{~cm}$ in size, ${ }^{\mathrm{a}} \chi^{2}=8.889, p<0.01 ;{ }^{\mathrm{c}} \chi^{2}=4.994, p<0.05$; compared with no lymph node metastasis, ${ }^{\mathrm{b}} \chi^{2}=10.020, p<0.01 ;{ }^{\mathrm{d}} \chi^{2}=7.166, p<0.01$.

in the well-differentiated cancer tissues than in the poorly-differentiated cancer tissues.

Tumor growth and metastasis are closely associated with tumor neoangiogenesis (Han et al. 2001). Tumor MVD is an effective index that reflects tumor angiogenesis, while CD34 is one of the best available markers to indicate the presence of blood vessel endothelial cells (Matsuyama et al. 1998; Mitsuhashi et al. 2003). The immunohistochemical examinations with a CD34 monoclonal antibody have confirmed that the level of MVD was closely correlated with the degree of cancer differentiation. In the present study, the MVD was significantly increased in the poorlydifferentiated adenocarcinoma than in the welldifferentiated adenocarcinoma. Therefore, we conclude that high MVD may indicate a poorer prognosis in colorectal adenocarcinoma.

Our study also demonstrated that the expression of Ang-1 and Ang-2 differentially correlated with the MVD. The MVD was significantly lower in Ang-1-expressing adenocarcinoma tissues, compared to Ang-1 negative tissues. In contrast, the MVD was significantly higher in
Ang-2-expressing tissues than in Ang-2 negative tissues. These findings indicate that the differential expression of Ang-1 and Ang-2 may play a crucial role in angiogenesis of the colorectal adenocarcinoma.

It has been suggested that Ang-1 and Ang-2 may mediate the stability of endothelial cells via binding to the tyrosine kinase Tie- 2 receptor. Through this binding, Ang-1 may enhance the adhesion of circumference blood vessels and stabilize neoangiogenesis, thereby limiting angiogenesis and tumor growth. In contrast, Ang-2 may enhance the sensitivity of endothelial cells to mitotic signals, and may promote angiogenesis, resulting in vessel instability and continuous tumor angiogenesis (Ellis et al. 2002).

Asahara et al. (1998) reported that Ang-2 may cooperate with VEGF in enhancing the formation of blood vessel net, thereby improving the recovery of damaged vessels and maintaining them in a plastic condition. Under such conditions, the blood vessels are likely to be more sensitive to VEGF signaling. The present study showed that the expression pattern of Ang-2 was 
similar to that of VEGF, and there was a significant correlation between Ang-2 and VEGF. Furthermore, our findings revealed that high expression of Ang-2 and VEGF may be closely related to tumor size and lymph-node metastases. A previous study from Etoh et al. (2001) suggested that in the presence of VEGF, Ang-2 could strongly up-regulate the production of endothelial cell matrix metalloproteinases (MMP-1, MMP-2) and urokinase fibrinolysin activator, subsequently leading to the continuous production of highly instable and immature tumor blood vessels with high endothelial cell proliferation capacity. This might be a reason for causing lymph-node metastases, revealing the critical role of Ang-2 in the process of tumor metastatic spread (Lobov et al. 2002; Yoshiji et al. 2005).

Based upon these findings, we hypothesize that in colorectal cancer tissues, the differential expression of Ang-1 and Ang-2 leads to enhance the activity of Ang-2. Namely, Ang-2, via Tie-2 and with the help of VEGF, is able to promote continuous neoagiogenesis, thereby leading to increased MVD levels. Our study provides further evidence that the Ang-Tie system might play a critical role in regulating angiogenesis in human colorectal carcinoma, and suggests that this system, especially Ang-2, may represent a novel target for cancer therapy. In fact, a novel antiangiogenesis strategy may decrease tumor development and metastasis (Pan and Tan et al. 2005).

\section{References}

Asahara, T., Chen, D., Takahashi, T., Fujikawa, K., Kearney, M., Magner, M., Yancopoulos, G.D. \& Isner, J.M. (1998) Tie2 receptor ligands, angiopoietin-1 and angiopoietin-2, modulate VEGF-induced postnatal neovascularization. Circ. Res., 83, 233-240.

Carvalho, J.F., Blank, M. \& Shoenfeld, Y. (2007) Vascular endothelial growth factor (VEGF) in autoimmune diseases. J. Clin. Immunol., 27, 246-256.

Ellis, L.M., Ahmad, S., Fan, F., Liu, W., Jung, Y.D., Stoeltzing, O., Reinmuth, N. \& Parikh, A.A. (2002) Angiopoietins and their role in colon cancer angiogenesis. Oncology, 16, 31-35.

Etoh, T., Inoue, H., Tanaka, S., Barnard, G.F., Kitano, S. \& Mori, M. (2001) Angiopoietin-2 is related to tumor angiogenesis in gastric carcinoma: possible in vivo regulation via induction of proteases. Cancer Res., 61, 2145-2153.
Hanahan, D. (1997) Signaling vascular morphogenesis and maintenance. Science, 277, 48-50.

Han, H., Silverman, J.F., Santucci, T.S., Macherey, R.S., d'Amato, T.A., Tung, M.Y., Weyant, R.J. \& Landreneau, R.J. (2001) Vascular endothelial growth factor expression in stage I non-small cell lung cancer correlates with neoangiogenesis and a poor prognosis. Ann. Surg. Oncol., 8, 72-79.

Katoh, Y. \& Katoh, M. (2006) Comparative integromics on Angiopoietin family members. Int. J. Mol. Med., 17, 1145-1149.

Leung, D.W., Cachianes, G., Kuang, W.J., Goeddel, D.V. \& Ferrara, N. (1989) Vascular endothelial growth factor is a secreted angiogenic mitogen. Science, 246, 1306-1309.

Lobov, I.B., Brooks, P.C. \& Lang, R.A. (2002) Angiopoietin-2 displays VEGF-dependent modulation of capillary structure and endothelial cell survival in vivo. Proc. Natl. Acad. Sci. USA, 99, 11205-11210.

Loughna, S. \& Sato, T.N. (2001) Angiopoietin and Tie signaling pathways in vascular development. Matrix Biol., 20, 319-325.

Maisonpierre, P.C., Suri, C., Jones, P.F., Bartunkova, S., Wiegand, S.J., Radziejewski, C. \& Compton, D. (1997) Angiopoietin-2, a natural antagonist for Tie-2 that disrupts in vivo angiogenesis. Science, 277, 55-60.

Matsuyama, K., Chiba, Y., Sasaki, M., Tanaka, H., Muraoka, R. \& Tanigawa, N. (1998) Tumor angiogenesis as a prognostic marker in operable non-small cell lung cancer. Ann. Thorac. Surg., 65, 1405-1409.

Mitsuhashi, N., Shimizu, H., Ohtsuka, M., Wakabayashi, Y., Ito, H., Kimura, F., Yoshidome, H., Kato, A., Nukui, Y. \& Miyazaki, M. (2003) Angiopoietins and Tie-2 expression in angiogenesis and proliferation of human hepatocellular carcinoma. Hepatology, 37, 1105-1113.

Pan, Y.L. \& Tan, L. (2005) Interleukin-12 inhibiting the expression of Ang-2 and VEGF gene in hepatocarcinoma of nude and its significance. Chin. J. Exp. Surg., 22, 439-441.

Saclatides, T.J. \& Speziale, N.J. (1994) Tumor angiogenesis and rectal carcinoma. Dis. Colon Rectum, 37, 921-926.

Weidner, N. (1995) Intratumor microvessel density as prognostic factor in cancer. Am. J. Pathol., 147, 9-19.

Wong, A.L., Haroon, Z.A., Werner, S., Dewhirst, M.W., Greenberg, C.S. \& Peters, K.G. (1997) Tie-2 expression and phosphorylation in angiogenic and quiescent adult tissues. Circ. Res., 81, 567-574.

Yancopoulos, G.D., Davis, S., Gale, N.W., Rudge, J.S., Wiegand, S.J. \& Holash, J. (2000) Vascular-specific growth factors and blood vessel formation. Nature, 407, 242-248.

Yoshiji, H., Kuriyama, S., Noguchi, R., Yoshii, J., Ikenaka, Y., Yanase, K., Namisaki, T., Kitade, M., Uemura, M., Masaki, T. \& Fukui, H. (2005) Angiopoietin 2 displays a vascular endothelial growth factor dependent synergistic effect in hepatocellular carcinoma development in mice. Gut, $\mathbf{5 4}$, 1768-1775.

Zadeh, G. \& Guha, A. (2003) Neoangiogenesis in human astrocytomas: expression and functional role of angiopoietins and their cognate receptors. Front. Biosci., 8, e128-e137.

Zhu, P. \& Chen, D.D. (2004) Expression of angiotensin-2 in gastric carcinoma and its relationship with clinically pathological features. Chin. J. Exp. Surg., 21, 440-441. 